\title{
Statistical Analysis of Determinants of Maternal Institutional Delivery Service Utilization in Ethiopia
}

\author{
Kasahun Takele Geneti \\ Department of Statistics, Haramaya University, Dire Dawa, Ethiopia
}

Email address:

kastake10@gmail.com

To cite this article:

Kasahun Takele Geneti. Statistical Analysis of Determinants of Maternal Institutional Delivery Service Utilization in Ethiopia. American Journal of Theoretical and Applied Statistics. Vol. 4, No. 3, 2015, pp. 71-77. doi: 10.11648/j.ajtas.20150403.11

\begin{abstract}
Utilization of maternal healthcare service is a proximate determinant of maternal morbidities and mortalities. On the way to improve maternal health care service, it is important to understand factors influencing maternal health care service utilization. The main purpose of this study is to determine statistically the correlates of maternal place of delivery in Ethiopia, using 2011 EDHS data. The overall frequency of maternal delivery at heath facility in Ethiopia was $12.8 \%$. Logistic regression model was used to model the effects of selected socioeconomic and demographic covariates. It was found that the covariates place of residence, birth order of child, mother's age at child birth, mother's educational level, household economic status and mothers employment status were the most important determinants of mothers place of delivery in the Ethiopia. It is suggested that to improve maternal healthcare service utilization, maternal education should be supported as a policy and this could be achieved through female literacy programs in the country.
\end{abstract}

Keywords: Place of Delivery, Determinants, Logistic Regression, Ethiopia

\section{Introduction}

Utilization of maternal healthcare services is a proximate determinant of maternal morbidities and mortalities. Maternal health has emerged as global priority because of a great gap in the status of mother's well being between the rich and the poor countries. According to WHO (2008), maternal health refers to the health of women during pregnancy, childbirth and the postpartum period. In rich nations, where women have access to basic health care, giving birth is a positive and fulfilling experience. On the other hand, for many women in poor countries it is associated with suffering, ill health and even death.

Complications of pregnancy and childbirth are a leading cause of maternal morbidities and mortalities for women of reproductive age ( $15-49$ years) in developing countries. The WHO estimates that over 500,000 women and girls die from complications of pregnancy and childbirth each year, worldwide, with approximately $99 \%$ of these deaths occurring in developing countries. The WHO report, 9000 maternal deaths occurred in Ethiopia in 2010. The 2011 Ethiopian Demographic and Health Survey (EDHS) showed that the maternal mortality ratio was 676 deaths per 100,000 live births. In other words, for every 1,000 live births, about seven women (6.76) died during pregnancy, child birth, or within two month s of child birth (WHO, 2012).

Internationally, increasing attention given to maternal health has been concentrated in reducing maternal mortality. The tragedy of not preventing these avoidable or treatable deaths resulted in 536,000 maternal deaths worldwide in 2005 (WHO, 2007). Developing regions accounted for 99 percent $(533,000)$ of these deaths, with sub-Saharan Africa and Southern Asia accounting for 86 percent of them (UN, 2008). Put another way, every minute of each year a woman dies from complications of pregnancy, abortion attempts and childbirth (UNFPA, 2004). Millions more women survive but suffer from illness and disability related to pregnancy and childbirth. It has been estimated by the Safe Motherhood Initiative (SMI) that 30 to 50 morbidities occur for each maternal death (SMI, 2003).

The utilization of maternal health care is one of the important factors to reduce the incidence of maternal mortality. United Nations reported recent data of maternal health care from developing countries are as follow: the number of pregnant women who receive at least one antenatal care is approximately 74 percent in 2005 (UN, 
2008); 40 percent of deliveries take place in health facilities and skilled health personnel assist nearly 61 percent of births in 2006 (UN, 2008). Obviously, the data indicates that the government commitment to maternal health care has not reached the levels required to make strong impact on mortality rates. Many existing interventions have been found to be ineffective in preventing maternal deaths. Laws and regulations in many countries sometimes impede health care policies (mostly in specific areas such as sexuality education and access of adolescents to reproductive health information and services).

Access to proper medical attention and hygienic conditions during delivery can reduce the risk of complications and infections that may lead to death or serious illness for the mother and/or baby (WHO, 2006). EDHS 2011 shows that 10 percent of women reported that their most recent live birth in the last five years was delivered by a health professional.

Despite the fact that using maternal health care services is essential for further improvement of maternal and child health, little is known about the current magnitude of use of and access to maternal care services. With the background given above this study aimed to fill the existing information gap about maternal health care by providing empirical evidence-based on the data of the 2011 EDHS by way of identifying factors that affect maternal healthcare services in Ethiopia.

\section{Data Source and Methodology}

\subsection{Data Source}

This study used the 2011 Ethiopian Demographic and Health Survey (EDHS) data set. The survey drew a representative sample of women of reproductive age, by administering a questionnaire and making an anthropometric assessment of women. The 2011 EDHS sample was selected using a stratified, two stage cluster design, and enumeration areas were the sampling units for the first stage. The sample included 624 enumeration areas, 187 in urban areas and 437 in rural areas. In the 2011 EDHS, information and data on delivery care was collected from women who had at least one birth in the five years before the survey. For the 2011 EDHS, representative samples of 17,817 households were selected. The analysis presented in this study on place of delivery was based on the 2087 women aged 15-49.

\subsection{Variables Used in the Study}

The outcome (response) variable for this study is place of delivery.

$$
\text { Place of delivery }=\left\{\begin{array}{l}
1 \text { if delivered in health facility } \\
0, \text { otherwise }
\end{array}\right.
$$

The explanatory variables which might determine place of delivery were place of residence, birth order of child, mother's age at child birth, mother's education level, household economic status (wealth index), and mother's employment status.

\subsection{Methods of Statistical Analysis}

Logistic regression analysis extends the techniques of multiple regression analysis to research situations in which the outcome variable is categorical. Logistic regression allows one to predict a discrete outcome, such as group membership, from a set of predictor variables that may be continuous, discrete, dichotomous, or a mix of any of these (Gellman and Hill, 2007).

Generally, if the dependent or response variable is dichotomous (binary), such as (alive or died, presence or absence, success or failure and etc) logistic regression is used. The logistic regression is also preferred from multiple regression and discriminate analysis as it results in a biologically meaningful interpretation, it is mathematically flexible and easily used distribution and it requires fewer assumptions (Hosmer and Lemeshow, 2000). Unlike the discriminate analysis, the logistic regression does not have the requirements of the independent variables to be normally distributed, linearly related, nor equal variance with in each group (Tabachnick and Fidel, 1996).

Logistic regression has a peculiar property of easiness to estimate logit differences for data collected both retrospectively and prospectively (Mc Cullagh and Nelder, 1983) have contributed a lot to its importance in application areas. There are two main uses of logistic regression: Firstly, to predict the group membership. Since logistic regression calculates the probability of success over the probability of failure, the results of the analysis are in the form of an odds ratio. Secondly, logistic regression also provides knowledge of the relationships and strengths among the variables.

\subsubsection{Model Description}

In this study since the response variable, that is place of delivery (home $=0$ or in health facility $=1$ ) is a dichotomous variable so the effect of explanatory variables on the dependent variable can be investigated using logistic regression model.

Let $\mathrm{Y}_{\mathrm{n} \times 1}$ be a dichotomous outcome random variable with categories 1 (delivery in health facility) and 0 (in home). Let $\mathrm{X}_{\mathrm{n} \times(\mathrm{p}+1)}$ denote the collection of $\mathrm{p}$ predicator variables of $\mathrm{Y}$. Then, the conditional probability that a mother's place of delivery given the $\mathrm{X}_{\mathrm{i}}$ set of predictor variables is denoted by $P\left(Y_{i}=1 / \mathbf{x}_{i}\right)=\pi_{i}$. The expression $\pi_{i}$ in logistic regression model can be expressed in the form of:

$$
\pi_{i}=\frac{e^{\beta_{0}+\beta_{1} x_{i 1}+\ldots .+\beta_{p} x_{i p}}}{1+e^{\beta_{o}+\beta_{1} x_{i 1}+\ldots+\beta_{p} x_{i p}}}=\frac{e^{X_{\mathbf{i}}^{\prime} \boldsymbol{\beta}}}{1+e^{X_{\mathrm{i}}^{\prime} \mathbf{\beta}}}=\frac{1}{1+e^{-X_{\mathbf{i}}^{\prime} \mathbf{\beta}}}
$$

Where:

$\pi_{i}=$ the probability of mother $i$ being delivered in health facility given $\mathrm{X}_{\mathrm{i}}$ set of predictor variables.

$Y_{i}=$ the observed mother's place of delivery in health facility/in home of mother $i$.

$\beta=(p+1) \times 1$ is a vector of unknown parameters including the constant. 
However, the relationship between the predictor and response variables is not a linear function in logistic regression; instead, the logarithmic transformation of equation yields the linear relationship between the predictor and response variables. So an alternative form of the logistic regression equation is the logit transformation of $\pi_{i}$ given as follows:

$$
\log i t\left(\pi_{i}\right)=\log \left(\frac{\pi_{i}}{1-\pi_{i}}\right)=\beta_{0}+\beta_{1} x_{i 1}+\ldots+\beta_{p} x_{i p}
$$

The coefficient can be interpreted as the change in the logodds associated with a one unit change in the corresponding independent variable or the odd increases multiplicatively by $e^{\beta}$ for every one unit change increase in $\mathbf{X}_{\mathbf{i}}$.

\subsubsection{Parameter Estimation for the Binomial Logistic Regression}

Suppose we have a sample of $n$ independent observations $\left\{\left(y_{i}, x_{i}\right)\right\}_{i=(1,2, \ldots, n)} \in\left(\{0,1\} \times \Re^{p+1}\right)^{n}$, where $y_{i}$ denotes the values of dichotomous outcome variable, and $x_{i}$ is the value of explanatory variables for the $i^{\text {th }}$ subject. Assume $y_{i} \sim$ Bernoulli $\left(1, \pi\left(x_{i}\right)\right), i=1,2, \ldots, n$. Based on the set of data, we estimate the parameter vector $\boldsymbol{\beta}=\left(\beta_{0}, \beta_{1}, \ldots, \beta_{p}\right)^{\prime} \in \mathfrak{R}^{p+1}$ to fit the logistic model in equation (2). To find the maximum likelihood estimator of $\beta$, we define the likelihood function as follows:

$$
\begin{aligned}
& L(\boldsymbol{\beta})=\prod_{i=1}^{n} \pi\left(x_{i}\right)^{y_{i}}\left(1-\pi\left(x_{i}\right)\right)^{1-y_{i}} \\
& =\prod_{i=1}^{n}\left(\frac{\pi\left(x_{i}\right)}{1-\pi\left(x_{i}\right)}\right)^{y_{i}}\left(1-\pi\left(x_{i}\right)\right) \\
& =\prod_{i=1}^{n}\left(\frac{\frac{e^{x_{i}^{\prime} \beta}}{1+e^{x_{i}^{\prime} \beta}}}{\frac{1+e^{x_{i}^{\prime} \beta}-e^{x_{i}^{\prime} \beta}}{1+e^{x_{i}^{\prime} \beta}}}\right)\left(\frac{1+e^{x_{i}^{\prime} \beta}-e^{x_{i}^{\prime} \beta}}{1+e^{x_{i}^{\prime} \beta}}\right) \\
& =\prod_{i=1}^{n} \frac{\left(e^{x_{i}^{\prime} \beta}\right)^{y_{i}}}{1+e^{x_{i}^{\prime} \beta}}=\prod_{i=1}^{n} \frac{e^{y_{i} x_{i}^{\prime} \beta}}{1+e^{x_{i}^{\prime} \beta}}
\end{aligned}
$$

Now, we find the maximum likelihood estimates, $\boldsymbol{\beta}$, of $\beta$ by maximizing the log-likelihood function for the observed values of $y_{i}$ and $\boldsymbol{x}_{\boldsymbol{i}}$. Since the log-likelihood equations are non- linear in the parameters, the Newton-Raphson iterative maximum likelihood estimation method that expresses $\hat{\beta}$ at the $(u+1)^{t h}$ cycle of the iteration is expressed as $\hat{\boldsymbol{\beta}}_{\mathbf{u}+\mathbf{1}}=\hat{\boldsymbol{\beta}}_{\mathbf{u}}+\left[\mathbf{X}^{\prime} \hat{\mathbf{V}}_{\mathbf{u}} \mathbf{X}\right]^{-1} \mathbf{X R}_{\mathrm{u}}$, where $u=0,1,2, \ldots$ and $\hat{\mathbf{V}}$ is a diagonal matrix with its diagonal elements $\mathbf{X}_{\mathbf{i}}=1$ ).
$\hat{\mathbf{V}}=\operatorname{diag}\left[\hat{\pi}_{i}\left(1-\hat{\pi}_{i}\right)\right]=\operatorname{cov}(\mathbf{y}) \cdot$ Finally, $\hat{\boldsymbol{\beta}}$ is the resultant maximum likelihood estimator of $\beta$ with residual $R=y_{i}-\hat{\pi}_{i}$ (Collet, 1991; Greene, 2003). Newton's method usually converges to the maximum of the log - likelihood in just a few iteration unless the data are especially badly conditioned (Greene, 2003). In fact all the parameters $\hat{\beta}_{o}, \hat{\beta}_{1}, \ldots, \hat{\beta} k$ and estimates of $\pi_{i}$ for each subject could be facilitated by the widely available statistical software package SPSS-20 with the latest version.

\subsubsection{Evaluations of a Logistic Regression Model}

A logistic model is said to provide a better fit to the data if it demonstrates an improvement over the intercept-only model (also called the null model, which has no predictors). The overall significance of coefficients of the logistic regression model will be accomplished using the Likelihood Ratio (LR) test (Hosmer and Lemeshow, 2000). It is performed on the basis of test statistic:

$$
G=-2\left[\ln \left(L_{0}\right)-\ln \left(L_{1}\right)\right]
$$

Where $\mathrm{L}_{0}$ is the likelihood of the null model and $\mathrm{L}_{1}$ is the likelihood of the saturated model. The statistic $G$ is distributed chi-squared with degrees of freedom equal to the difference in the number of degrees of freedom between the two models. If this difference is statistically significant, then the less restrictive model (the one with more variables) is said to fit the data significantly better than the more restrictive model.

The statistical significance of individual regression coefficients will be tested using the Wald and Score chisquare statistic. The Wald statistic is a test which is commonly used to test the significance of the individual logistic regression coefficients for each independent variable (that is, to test the null hypothesis in logistic regression that a particular logit (effect) coefficient is zero i.e. $H_{0}: \beta_{i}=0$ against $\beta_{i} \neq 0$.

For a dichotomous dependent variable, the Wald statistic is:

$$
W=\left[\frac{\hat{\beta_{i}}}{S E\left(\hat{\beta}_{i}\right)}\right]^{2}
$$

Under the null hypothesis for large sample size, this statistic has an approximate chi-square distribution with one degree of freedom.

One primary goal of performing logistic regression is to generate an equation that can reliably classify observations into one of two outcomes. The degree to which predictions agree with the data may be shown graphically by either a receiver operating characteristic (ROC) curve or an overlay plot of sensitivity and specificity versus predicted probabilities (Afifi \& Clark, 1990; Hosmer \& Lemeshow, 2000). This paper used ROC curve to see the degree of 
agreement between the actual and predicted values.

\section{Results and Discussion}

\subsection{Descriptive Analysis}

The aim of this study was to determine the differential factors that influence utilization of mothers' health care service in Ethiopia. The distribution of mothers who had a live birth in the five years preceding the survey by utilization of place of delivery for the most recent birth is given in Table 1.

Utilization of the service/ institutional delivery was $62.0 \%$ for older mothers, $71.9 \%$ for younger mothers and $27.8 \%$ for adolescent mothers. For the birth order one to third and four and higher, respectively, were $32.2 \%, 62.3 \%$. Institutional delivery was $83.2 \%$ for urban residences and $46.8 \%$ for rural residences.

As can be seen from Table1, institutional delivery was $39.2 \%$ for poor mothers, $44.9 \%$ for middle income mothers and $66.9 \%$ for rich mothers. For the unemployed and employed mothers, respectively, were $50.5 \%$ and $56.7 \%$.

In this study, mother's educational attainment recorded into three categories as no formal education, primary education and secondary and above. From Table 1, mother's place of delivery varies by their educational attainment. Institutional delivery was $35.5 \%$ for no formal education, $67.5 \%$ for primary education and $95.5 \%$ for secondary and above mother's educational attainment.

Table 1. Distribution of Socio-economic and demographic characteristics of maternal health care services utilization in Ethiopia.

\begin{tabular}{|c|c|c|c|c|c|}
\hline \multirow{3}{*}{ Variables } & \multirow{3}{*}{ Level } & \multicolumn{4}{|c|}{ Place of delivery } \\
\hline & & \multicolumn{2}{|c|}{ Home } & \multicolumn{2}{|c|}{ Health Center } \\
\hline & & Frequency & Percent & Frequency & Percent \\
\hline \multirow{3}{*}{ Mother's age in years } & $15-24$ & 244 & 28.1 & 623 & 71.9 \\
\hline & $25-35$ & 575 & 72.2 & 221 & 27.8 \\
\hline & $36-49$ & 161 & 38.0 & 263 & 62.0 \\
\hline \multirow{2}{*}{ place of residence } & Urban & 60 & 16.8 & 298 & 83.2 \\
\hline & Rural & 920 & 53.2 & 809 & 46.8 \\
\hline \multirow[t]{2}{*}{ Educational attainment } & primary education & 316 & 32.5 & 655 & 67.5 \\
\hline & secondary and higher & 4 & 4.3 & 88 & 95.7 \\
\hline \multirow{3}{*}{ Wealth index } & Poor & 433 & 60.8 & 279 & 39.2 \\
\hline & Middle & 226 & 55.1 & 184 & 44.9 \\
\hline & Rich & 321 & 33.3 & 644 & 66.7 \\
\hline \multirow{2}{*}{ Birth order number } & $1-3$ & 437 & 67.8 & 208 & 32.2 \\
\hline & $4 \&$ above & 543 & 37.7 & 899 & 62.3 \\
\hline
\end{tabular}

\subsection{Determinants of Mothers Place of Delivery in Ethiopia}

The relationship between mothers' age at child birth and the dependent variable place of delivery is significant; mothers of age at child birth $15-24$ years were 1.636 times more likely to deliver at health facility than those mothers' age at child birth 36-49 years. Mothers age at child birth 2535 years were 0.207 times less likely to deliver at health facility than those $36-49$ years old.
Mothers' educational attainment has a significant contribution on mother's place of delivery. Mothers whose educational attainment is primary were 0.082 times less likely to deliver at health facility than those who have secondary and above education. Mothers of no formal education were 0.036 less likely to deliver at health facility than those who have secondary and above education.

Table 2. Logistic Regression Analysis of the Determinants of maternal health care services utilization in Ethiopia.

\begin{tabular}{|c|c|c|c|c|c|c|c|}
\hline Variable & Level & B & S.E. & Wald & Df & P-value & $\operatorname{Exp}(B)$ \\
\hline \multirow[t]{3}{*}{ Mother's age at birth in years $(\operatorname{Ref}(36-49))$} & & & & 24.964 & 2 & .000 & \\
\hline & $15-24$ & .492 & .162 & 9.202 & 1 & .002 & 1.636 \\
\hline & $25-35$ & -1.573 & .148 & 112.844 & 1 & .000 & .207 \\
\hline Place of residence (Urban) & Urban & 1.858 & .211 & 77.574 & 1 & .000 & 6.410 \\
\hline $\begin{array}{l}\text { Mother's education (Ref (secondary and } \\
\text { above)) }\end{array}$ & & & & 66.486 & 2 & .000 & \\
\hline \multicolumn{8}{|l|}{ Ref (secondary and above) } \\
\hline No formal education & No formal education & -3.318 & .574 & 33.448 & 1 & .000 & .036 \\
\hline primary education & primary education & -2.505 & .571 & 19.237 & 1 & .000 & .082 \\
\hline wealth Index (Ref (Rich)) & & & & 12.778 & 2 & .002 & \\
\hline Poor & Poor & -.488 & .137 & 12.748 & 1 & .000 & .614 \\
\hline Middle & Middle & -.244 & .155 & 2.462 & 1 & .117 & .784 \\
\hline Birth order (4 \& above) (Ref (1-3)) & $4 \&$ above & -1.783 & .136 & 17.844 & 1 & .000 & .168 \\
\hline Mother's employment ( Ref(employed)) & Unemployed & -.264 & .114 & 5.348 & 1 & .021 & .768 \\
\hline
\end{tabular}


Mothers whose place of residence is urban area were 6.410 times more likely to deliver at health facility than whose place of residence is rural controlling for other variables in the model. The relationship between wealth index and place of delivery was significant. The estimated odds ratios for mothers in the categories of wealth indices poor and middle compared to those mothers in the rich category were 0.614 and 0.784 respectively.

The relationship between birth order and Place of Delivery was also found to be significant. Mothers who experienced 4 and above birth order were 0.168 times less likely to deliver at health facility than those who experienced 1-3 birth order number. Unemployed mothers were 0.768 times less likely to deliver at health facility than employed mothers.

\subsection{Assessing the Fit of the Model}

The ROC curve is a plot of the true positive rate (sensitivity) against the false positive rate (1-specificity) for the different possible cut-offs of a diagnostic test. The area under the ROC curve, which ranges from zero to one, provides a measure of the model's ability to discriminate between those subjects who experience the outcome of interest (delivery in health facility) versus those who do not (home). The results of goodness-of-fit can therefore be summed up in a single number: the area under the ROC curve (often abbreviated to AUC). The model with a larger area below the ROC curve (i.e., a larger AUC statistic) is considered better model (Afifi \& Clark, 1990). Besides if 0.8 $\leq \mathrm{AUC}<0.9$ this is considered excellent discrimination (Hosmer and Lemeshow, 2000). In other words, the best model is the one associated with the greatest sensitivity and the lowest 1 - specificity. In our case the AUC is 0.8562 as we can see in the following graph. This implies that the model fit the data very well.

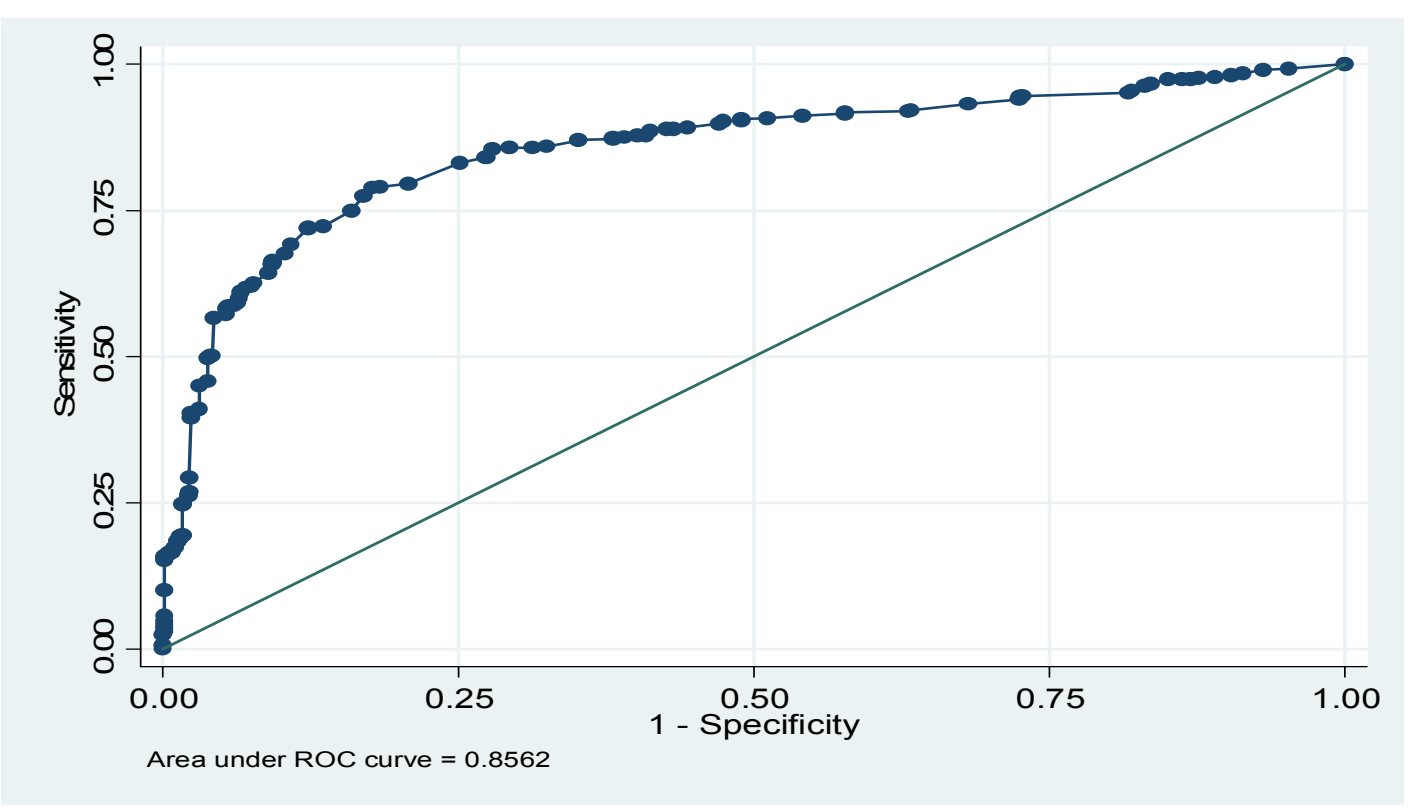

Figure 1. Receiver Operating Characteristics (ROC)

\subsection{Discussion}

This study was premeditated to identify the associated factors of maternal place of delivery in Ethiopia based on EDHS 2011 data. Accordingly, Logistic regression analysis on place of delivery was employed to identify the effect of covariates on maternal place of delivery. The results obtained are discussed as follows. The total number of women presented in this study was 2087 whose age 15-49, among which $11.3 \%$ were deliver at health facility. The Logistic regression analysis revealed that the covariates: place of residence, birth order of child, mother's age at child birth, mother's education level, household economic status (wealth index), and mothers employment status were the most important determinants of mothers institutional delivery in Ethiopia.

Mothers' educational attainment has a significant contribution on mother's place of delivery. Mothers whose educational attainment is primary were 0.082 times less likely to deliver at health facility than those who have secondary and above education. Mothers of no formal education were 0.036 less likely to deliver at health facility than those who have secondary and above education. This could be attributed to the fact that women or mothers with no or low educational attainment are unable to inculcate modern health knowledge and practices which are basic requirements for enhancing mothers health. Women who are educated are better able to break away from traditional practices to use modern health care services in order to enhance their health, educated women are better able to make use of and turn to their advantage what is available in their community, educated women may be more empowered which will enhance their decision-making power and improve their ability for making independent decisions on their health 
leading to greater modern healthcare utilization. This is consistent with the findings of (ESPS, 2008, Kamal, 2009, Kawahara, 2010) that health care utilization is lower among women with primary education and lower among women with higher education.

This study found a significant relationship between birth order and place of delivery. This is similar to other studies reported elsewhere (Kamal, 2009, Asfawosen A.et al, 2014). Women having their first babies were more likely to utilize institutional delivery than women with higher order births. Having more children is usually associated with increased physical and material responsibilities which could leave women with very little time and financial resources to care for themselves. This could discourage the use of mother's institutional delivery.

Strong association has been found between household wealth index and the use of maternal health services which is also supported by other studies (Babalola, 2009, Ntambue et al, 2012). This can be due to the fact that women should be able to cover the costs needed in order to access health care services. Even in areas where maternal health care services are provided for free, women still have to pay for transportation and additional costs. As a result only those women who can afford to pay for such costs are able to visit health facilities.

Place of residence has been found to be significantly associated with maternal health care service utilization in several studies (Ntambue et al, 2012, Shiferaw et al, 2013, Teferra et al, 2012). Consistent results were obtained in this study. The differences in utilization of maternal health care service between the urban and rural areas could be due to differences in availability and accessibility of maternal health care facilities. Usually, these facilities are disproportionately distributed in favor of urban areas in Ethiopia.

The study found a strong relationship between employment and institutional delivery utilization in Ethiopia. Unemployed mothers were 0.768 times less likely to deliver at health facility than employed mothers. The fact that employed women are more likely to utilize maternal health care service in Ethiopia gives an indication that the earning ability of women is an important factor for utilization; as found by other studies (Asfawosen A.et al, 2014, Teferra et al, 2012).

The relationship between mothers' age at child birth and the dependent variable place of delivery is significant; younger mothers more likely to deliver at health facility than those older mothers. This finding is consistent with some studies (Shiferaw et al, 2013, Ntambue et al, 2012). The low level of utilization recorded among older women may be due to increased experience in pregnancy and childbearing leading to the belief that they no longer need the assistance of a professional, especially if they have had no complications.

\section{Conclusion and Recommendations}

The study identifies the important predictors of maternal place of delivery in Ethiopia using Logistic regression model.
It employed the nationally representative data from the Ethiopian Demographic and Health Survey (EDHS, 2011) data. The study revealed that socio-economic and demographic variables have significant effect on the maternal place of delivery in Ethiopia. Using Logistic regression model the predictors: place of residence, birth order of child, mother's age at child birth, mother's education level, household economic status (wealth index), and mothers employment status were the most important determinants of mothers institutional delivery in the country. Mothers' educational attainment has a significant effect on mother's place of delivery. Mothers whose educational attainment is secondary and above were more likely to deliver at health facility than those who have no formal education and primary education. Moreover, the study revealed that younger mothers more likely to deliver at health facility than those older mothers. Furthermore, the finding showed that unemployed mothers were less likely to deliver at health facility than employed mothers in Ethiopia. In the light of the above conclusions, the study would recommend that effort should be made for providing better access to education, health facilities and creating job opportunity for mothers so that the gap in maternal place of delivery is bridged.

\section{Acknowledgment}

I sincerely acknowledge Haramaya University (one of the known, oldest and public university in Ethiopia) for allowing me to undertake this study with a great encouragement and financial support. My heartfelt appreciation also extends to Central Statistical Agency (CSA) of Ethiopia for providing me necessary materials and data.

\section{References}

[1] Afifi, A.A. \& Clark, V. (1990). Computer Aided Multivariate Analysis, $2^{\text {nd }}$ Edition. New York: Van Nostrand Reinhold.

[2] Asfawosen A.et al (2014). Factors Associated with Maternal Health Care Services in Enderta District, Tigray, Northern Ethiopia: A Cross Sectional Study. American Journal of Nursing Science. Vol. 3, No. 6, pp. 117-125. doi: 10.11648/j.ajns.20140306.15

[3] Babalola S, Fatusi A(2009). Determinants of use of maternal health services in Nigeria, looking beyond individual and household factors. BMC Pregnancy Childbirth.

[4] Central Statistical Agency [Ethiopia] and ICF International. 2011. Ethiopia Demographic and Health Survey 2011. Addis Ababa, Ethiopia and Calverton, Maryland, USA: Central Statistical Agency and ICF International.

[5] Collet, D. (2003). Modeling Binary Data. Chapman \& Hall, London.

[6] Ethiopian Society of Population Studies (2008). Maternal healthcare seeking behavior in Ethiopia: Findings from EDHS 2005. Addis Ababa, Ethiopia.

[7] Gellman, A. and Hill, J. (2007). Data Analysis Using Regression and Multilevel or Hierarchical Models. Cambridge University Press, Columbia University. 
[8] Greene, W. (2003). Econometric Analysis, $5^{\text {th }}$ edition. Upper Saddle River, New Jersey

[9] Hosmer, D. and Lemeshow, S. (2000). Applied Logistic Regression. John Wiley and Sons, Inc., Newyork.

[10] Kamal, S.M.M. (2009). Factors Affecting Utilization of Skilled Maternity Care Services Among Married Adolescents in Bangladesh, Asian Population Studies, PP.153-170.

[11] McCullough, P. and Nelder, J. A. (1983). Generalized Linear Models. Chapman \& Hall, New York.

[12] Ntambue et al (2012):: Determinants of maternal health services utilization in urban settings of the Democratic Republic of Congo: A Case study of Lubumbashi City. BMC Pregnancy and Childbirt.

[13] Shiferaw et al (2013).Why do women prefer home births in Ethiopia? BMC Pregnancy and Childbirth

[14] Tabachnick, B. and Fidell, L. (1996). Using Multivariate Statistics, ${ }^{\text {rd }}$ edition. Harper Collins.
[15] Teferra AS, Alemu FM, Woldeyohannes SM (2012): Institutional delivery service utilization and associated factors among mothers who gave birth in the last 12 months in Sekela District, North West of Ethiopia: a community-based cross sectional study. BMC Pregnancy Childbirth

[16] United Nations (2008). The Millennium Development Goals Report, New York.

[17] WHO (2012). Trends in maternal mortality: 1990 to 2010 Estimates developed by WHO, UNICEF, UNFPA and The World Bank.

[18] UNFPA (2004): State of the World's Population. New York: Maternal Health.

[19] WHO (2008), Maternal Health, viewed 2 November 2008, Available from: URL:http://www.who.int/topics/maternal health/en/

[20] World Health Organization (2007). Maternal mortality in 2005: estimates developed by WHO, UNICEF, UNFPA, and the World Bank. Geneva: WHO. 